\title{
A formal system of first-order predicate calculus with infinitely long expressions
}

\author{
By Shôji MAehara and Gaisi TAKeuti
}

(Received April 3, 1961)

The first-order predicate calculus with infinitely long expressions is being developed by Berkeley school. We shall give a formal system for this calculus and prove a "Main Theorem" which implies the completeness theorem in Gödel's sense, and the cut-elimination theorem, Löwenheim-Skolem theorem and Craig's interpolation theorem.

Our formal system will be obtained from Gentzen's calculus $L K^{1)}$ by extending the concepts 'formula' and 'sequent', and by moderating the restriction on eigenvariables. This moderation of the restriction on eigenvariables plays an important rôle in making our system complete. We shall first explain by way of introduction, our moderation of the restriction on eigenvariables in case of $L K$.

One of the authors had a good chance to make a sojourn in Berkeley in 1960 where he could attend the seminar on this subject. He wishes to express his thankfulness to Professors Tarski, Henkin and their colleagues, for their kindness, and especially to Prof. Dana Scott for his stimulating discussions.

\section{$\S 1$. A moderation on the restriction on eigenvariables in the ordinary predicate calculus.}

In the Gentzen calculus $L K$, the restriction on eigenvariables is stated as follows :

The eigenvariable of an inference 'introduction of $\forall$ in succedent' or 'introduction of $\exists$ in antecedent' shall not occur in its conclusion.

Now we shall consider the new calculus obtained from $L K$ by replacing the restriction on eigenvariables by the following one:

Every formal proof satisfies the following three conditions:

1) The principal formulae of inferences which have a same eigenvariable are all one and the same formula.

2) For each free varable, a non-negative integer named the height can be

1) Cf. Gentzen [2]. English terminologies on $L K$ will be used, for most part, according to the usage in Kleene's text book [6]. 
uniquely defined as follows :

The height of the free variable occurring in an inference as the eigenvariable is $n+1$, where $n$ is the greatest number of the heights of those free variables which are contained in the principal formula. If a free variable does not occur in any inference as the eigenvariable, the height of the free variable is 0 .

3) Each variable occuring in an inference as the eigenvariable shall not occur in the endsequent.

This can be shown as follows. By Hilbert-Bernays' second $\varepsilon$-theorem ${ }^{2)}$, we have only to prove that our endsequent is provable in the $\varepsilon$-calculus obtained from $L K$ by adding $\varepsilon$-terms and ' $\varepsilon$-axioms' of the form

$$
\mathfrak{A}(t) \rightarrow \mathfrak{P}(\varepsilon x \mathfrak{Y}(x)),
$$

where $t$ is an arbitary term and $\varepsilon x \mathfrak{A}(x)$ is an arbitary $\varepsilon$-term. To prove this, we have only to substitute an appropriate $\varepsilon$-term for each free variable occuring as the eigenvariable of an inference throughout our formal proof and add $\varepsilon$-axioms adequately.

\section{$\S 2$. System of the first-order predicate calculus with infinitely long ex- pressions.}

In what follows, we always assume the axiom of choice, and consider a fixed strongly inaccessible number $\Omega_{0}$.

1. Formulae in our calculus are formed from simpler ones by means of the following operations:

1) the formation of the negation $7 \mathfrak{A}$ of a formula $\mathfrak{A}$;

2) the formation of the disjunction $\vee\left[\left\{\mathfrak{A}_{\lambda}\right\}_{\lambda<\alpha}\right]$ simply denoted by $\vee \mathfrak{A}_{\lambda}$, of a finite or transfinite well-ordered sequence of formulae $\left\{\mathfrak{H}_{\lambda}\right\}_{\lambda<\alpha}\left(\alpha<\Omega_{0}\right)$;

3) the existential quantification

$$
\left(\exists x_{0} x_{1} \cdots x_{\lambda} \cdots\right) \mathfrak{R}\left(x_{0}, x_{1}, \cdots, x_{\lambda}, \cdots\right)
$$

of a formula $\mathfrak{A}\left(a_{0}, a_{1}, \cdots, a_{\lambda}, \cdots\right)$ over a finite or transfinite well-ordered sequence of distinct free variables $\left\{\alpha_{\lambda}\right\}_{2<\alpha}$, and with respect to a sequence of distinct bound variables $\left\{x_{\lambda}\right\}_{\lambda<\alpha}\left(\alpha<\Omega_{0}\right)$.

Prime formulae in our case consist of a predicate symbol followed by a finite or transfinite well-ordered sequence of terms whose ordinal type is less than $\Omega_{0}$, where terms mean free variables, as long as no function symbols are used.

Small German letters stand for well-ordered sequences of terms or of distinct variables. For example, if $t$ stands for a sequence of terms $t_{0}, t_{1}, \cdots$, and

2) Cf. Hilbert-Bernays [4]. 
$\mathfrak{x}$ stands for a sequence of bound variables $x_{0}, x_{1}, \cdots$, then $\mathfrak{P}(\mathfrak{l})$ means $\mathfrak{A}\left(t_{0}, t_{1}, \cdots\right)$ and $\exists \mathfrak{x} \mathfrak{R}(\mathfrak{x})$ means $\left(\exists x_{0} x_{1} \cdots\right) \mathfrak{R}\left(x_{0}, x_{1}, \cdots\right)$.

Sequents in our calculus are formal expressions of the form $\Gamma \rightarrow \Theta$, where each of $\Gamma$ and $\Theta$ is an arbitary well-ordered sequence of zero or more formulae whose ordinal type is less than $\Omega_{0}$.

2. Postulates for the PREdicate Calculus With infinitely long ExPRESSIONS.

2.1. Axiom schema.

$$
\mathfrak{D} \rightarrow \mathfrak{D}
$$

2.2. Rules of inference.

2.21. Logical rules of inference:

2.211. Introduction of 7 in succedent :

$$
\begin{aligned}
& \left\{\mathfrak{A}_{\lambda}\right\}_{\lambda<\alpha}, \Gamma \rightarrow \Theta \\
& \Gamma \rightarrow \Theta,\left\{7 \mathfrak{A}_{\lambda}\right\}_{\lambda<\alpha}
\end{aligned}
$$

2.212. Introduction of 7 in antecedent:

$$
\frac{\Gamma \rightarrow \Theta,\left\{\mathfrak{A}_{\lambda}\right\}_{\lambda<\alpha}}{\left\{7 \mathfrak{A}_{\lambda}\right\}_{\kappa<\alpha}, \Gamma \rightarrow \Theta} ;
$$

2.213. Introduction of $\vee$ in succedent :

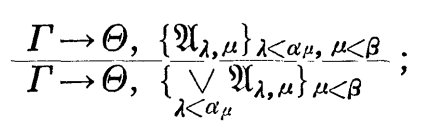

2.214. Introduction of $\vee$ in antecedent:

$$
\frac{\left\{\mathfrak{A}_{\lambda \mu, \mu}\right\}_{\mu<\beta}, \Gamma \rightarrow \Theta \text { for all }\left\{\lambda_{\mu}\right\}_{\mu<\beta} \text { such that } \lambda_{\mu}<\alpha_{\mu}(\mu<\beta)}{\left\{\underset{\lambda<\alpha_{\mu}}{\vee} \mathfrak{H}_{\lambda, \mu}\right\}_{\mu<\beta}, \Gamma \rightarrow \Theta} ;
$$

2.215. Introduction of $\exists$ in succedent :

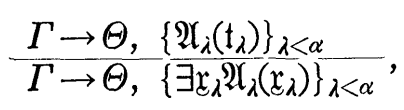

where t's are sequences of arbitrary terms;

2.216. Introduction of $\exists$ in antecedent:

$$
\frac{\left\{\mathfrak{H}_{\lambda}\left(\mathfrak{a}_{\lambda}\right)\right\}_{\lambda<\alpha}, \Gamma \rightarrow \Theta}{\left\{\exists \mathfrak{x}_{\lambda} \mathfrak{A P}_{\lambda}\left(\mathfrak{x}_{\lambda}\right)\right\}_{\lambda<\alpha}, \Gamma \rightarrow \Theta},
$$

where $\mathfrak{a}$ 's are sequences of distinct free variables. Each variable occurring in $\mathfrak{a}$ 's is called an eigenvariable of the inference. When an eigenvariable of that inference occurs in $\mathfrak{a}_{\lambda}, \exists \mathfrak{x}_{\lambda} \mathfrak{A}_{\lambda}\left(\mathfrak{x}_{\lambda}\right)$ is called the principal formula of the eigenvariable and $\mathfrak{I}_{\lambda}\left(\mathfrak{a}_{\lambda}\right)$ is called the side formula of the principal formula. When $\mathfrak{a}_{\lambda}=$ $\left\{a_{\lambda, \mu}\right\}_{\mu<\beta_{\lambda}}, \mu$ is called the order of the eigenvariable $\alpha_{\lambda, \mu}$ with respect to the principal formula $\exists \mathfrak{x}_{\lambda} \mathfrak{I}_{\lambda}\left(\mathfrak{x}_{\lambda}\right)$.

2.22. Structural rule of inference: 


$$
\frac{\Gamma \rightarrow \Theta}{\Delta \rightarrow \Lambda},
$$

where any formula occurring in $\Gamma$ or $\Theta$ is contained in $\Delta$ or $\Lambda$, respectively.

2.23. Cut :

$$
\frac{\Gamma \rightarrow \Theta, \mathfrak{D} \quad \mathfrak{D}, \Delta \rightarrow \Lambda}{\Gamma, \Delta \rightarrow \Theta, \Lambda} .
$$

\section{RESTRICTION ON EIGENVARIABLES.}

Every formal proof satisfies the following three conditions:

3.1. If a free variable occurs in two or more inferences as eigenvariables, the principal formulae of these eigenvariables are all one and the same formula, and the order of these eigenvariables with respect to each principal formula does not depend on inferences.

3.2. For each free variable, an ordinal number named the height can be uniquely defined as follows:

The height of the free variable occurring in an inference as an eigenvariable is $\nu+1$, where $\nu$ is the supremum of the heights of those free variables which are contained in the principal formula of that eigenvariable. If a free variables does not occur in any inference as an eigenvariable, the height of the free variable is 0 .

3.3. Each variable occurring in an inference as an eigenvariable shall not occur in the endsequent.

4. EXAMPLE OF A FORMAL PROOF (cf. Karp [5]). In the following, the conjunction symbol $\wedge$ and the universal quantifer $\forall x$ are regarded as abbreviations of $7 \vee 7$ and $7 \exists x>$, respectively.

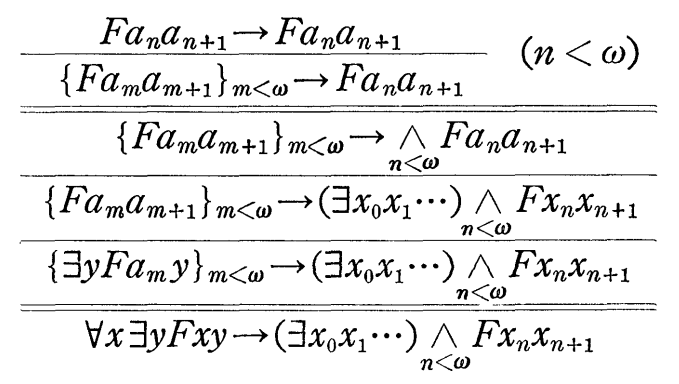

\section{$\S 3$. Main theorem, completeness theorem and cut-elimination theorem.}

Our main theorem on the predicate calculus with infinitely long expressions is regarded as a generalization of Gödel's completeness theorem (cf. Gödel [3]) and Gentzen's cut-elimination theorem (cf. Gentzen [2]).

MAIN THEOREM. Every sequent which is valid in any non-empty domain is provable without cut.

As a corollary of the main theorem, we have the following 
COMPLETENESS THOREM. ${ }^{3)}$ Every sequent which is valid in any non-empty domain is provable.

In the proof of the main theorem, we shall use an auxiliary $\varepsilon$-calculus with infinitely long expressions.

We shall call it simply ' $\varepsilon$-calculus'. The calculus in $\S 2$ will be called ' $\varepsilon$-less calculus'

1. In our $\varepsilon$-calculus, the $\varepsilon$-terms defined as follows, are also treated as terms. An $\varepsilon$-term $(\varepsilon \mathfrak{x})^{\lambda \mathfrak{H}(\mathfrak{x})}$ is formed from a formula $\mathfrak{T}(\mathfrak{a})$ with $\mathfrak{a}=\left\{\alpha_{\lambda}\right\}_{\lambda<\boldsymbol{x}}$ and an ordinal number $\lambda$ by the help of a sequence $x$ of bound variables $\left(\lambda<\alpha<\Omega_{0}\right)$. By $\varepsilon_{\mathfrak{C}} \mathfrak{R}(\mathfrak{x})$ we mean the sequence $\left\{(\varepsilon \mathfrak{x})^{\lambda} \mathfrak{I}(\mathfrak{x})\right\}_{\lambda<x}$, which indicates informally, such an $\mathfrak{x}$ as makes $\mathfrak{N}(\mathfrak{r})$ true, if it exists. Otherewise $\varepsilon \mathfrak{r} \mathfrak{\mathfrak { T }}(\mathfrak{r})$ means an arbitrary sequence of individuals whose ordinal type is $\alpha$.

We shall use, in the $\varepsilon$-calculus, the postulates for the $\varepsilon$-less calculus as they are, except the introduction of $\exists$ in antecedent. As infereneces 'introduction of $\exists$ in antecedent' we use those of the following form only:

$$
\frac{\left\{\mathfrak{R}_{\lambda}\left(\varepsilon_{\lambda} \mathfrak{x}_{\lambda}\left(\mathfrak{x}_{\lambda}\right)\right)\right\}_{\lambda<\alpha}, \Gamma \rightarrow \Theta}{\left\{\exists \mathfrak{x}_{\lambda} \mathfrak{P I}_{\lambda}\left(\mathfrak{x}_{\lambda}\right)\right\}_{\lambda<\alpha}, \Gamma \rightarrow \Theta}
$$

Accordingly, the concept of eigenvariable is not used in the $\varepsilon$-calcnlus, so we have no further use of the restriction on eigenvariables.

We have the following theorems on the $\varepsilon$-calculus and the $\varepsilon$-less calculus.

THEOREM 1. If a sequent is provable in the $\varepsilon$-less calculus, then the sequent is provable in the e-calculus.

This theorem is easily proved by substituting, in the $\varepsilon$-less formal proof of the concerned sequent, $\varepsilon$-terms for all free variables occurring as eigenvariables (cf. $\S 1$ ). By Theorem 1, we have the following result concernig the $\varepsilon$ less calculus :

THEOREM 2. Every sequent which is provable in the e-less calculus is valid in every non-empty domain.

By this theorem, as a corollary of the main theorem, we have the following

CUT-Elimination THEOREM. If a sequent is provable in the e-less calclus, then it is provable without cut in the same calculus.

The following Theorem 3 can be proved by substituting appropriate free variables for all $\varepsilon$-terms in formal proofs.

THEOREM 3. If an $\varepsilon$-less sequent is provable without cut in the e-calculus, then the sequent is provable without cut in the E-less calculus.

2. For the proof of our main theorem, it is sufficient, by Theorem 3, to prove the following

3) D. Scott and A. Tarski had proved, in [9], the completeness theorem on a. system for the propositional calculus with infinitely long expressions. 
THEOREM 4. Every e-less sequent which is valid in any non-empty domain and which is formed by formation rules in the e-calculus is provable without cut in the e-calculus.

The rest of this section is devoted to the proof of Theorem 4.

This proof will be carried through in following the idea of Schütte [8].

First of all, for each sequent $\subseteq$ in the $\varepsilon$-calculus, we consider the minimal genealogical-tree-formed figure $\boldsymbol{P}(\mathbb{S})$ consisting of sequents which satisfies the following conditions 1) -5) :

1) The lowermost sequent is $\mathbb{S}$.

2) When a sequent $\Delta \rightarrow \Lambda$ contains at least one formula whose outermost logical symbol is 7 , the immediate ancestor of $\Delta \rightarrow \Lambda$ is the sequent

$$
\left\{\mathfrak{B}_{\mu}\right\}_{\mu<\beta}, \Gamma \rightarrow \Theta,\left\{\mathfrak{H}_{\lambda}\right\}_{\lambda<\alpha},
$$

where $\left\{>\mathfrak{A}_{\lambda}\right\}_{\lambda<x}$ and $\left\{>\mathfrak{B}_{\mu}\right\}_{\mu<\beta}$ are the sequences of all formulae in $\Delta$ and in $\Lambda$, respectively, whose outermost logical symbol is 7 , and $\Gamma$ and $\Theta$ are the results obtained from $\Delta$ and from $\Lambda$, respectively, by suppressing all the formulae $7 \mathfrak{A}$ and $7 \mathfrak{B}$.

3) When a sequent $\Delta \rightarrow \Lambda$ contains no formula whose outermost logical symbol is 7 , and contains at least one formula whose outermost logical symbol is $V$, all the immediate ancestors of $\Delta \rightarrow \Lambda$ are the sequents

$$
\left\{\mathfrak{P}_{\lambda_{\mu}, \mu}\right\}_{\mu<\beta}, \Gamma \rightarrow \Theta,\left\{\mathfrak{B}_{\rho, \sigma}\right\}_{\rho<r_{\sigma}, \sigma<\delta} \quad\left(\lambda_{\mu}<\alpha_{\mu}\right),
$$

where $\left\{\underset{\lambda<\alpha_{\mu}}{\vee} \mathfrak{A}_{\lambda, \mu}\right\}_{\mu<\beta}$ and $\left\{\underset{\rho<\gamma_{\sigma}}{\vee} \mathfrak{B}_{\rho, \sigma}\right\}_{\sigma<\delta}$ are the sequences of all formulae in $\Delta$ and in $\Lambda$, respectively, whose outermost logical symbol is $\vee$, and $\Gamma$ and $\Theta$ are the results obtained from $\Delta$ and from $\Lambda$, respectively, by suppressing all the formulae $\vee \mathfrak{A}$ and $\vee \mathfrak{B}$.

4) When a sequent $\Delta \rightarrow \Lambda$ contains no formula whose outermost logical symbol is 7 or $\vee$, and contains at least one formula whose outermost logical symbol is $\exists$, the immediate ancestor of $\Delta \rightarrow \Lambda$ is the sequent

$$
\left\{\mathfrak{H}_{\lambda}\left(\varepsilon_{\mathfrak{L}_{\lambda}} \mathfrak{I}_{\lambda}\left(\mathfrak{x}_{\lambda}\right)\right)\right\}_{\lambda<x}, \Gamma \rightarrow \Theta,\left\{\mathfrak{B}_{\mu}\left(\mathrm{t}_{\mu, \rho_{\mu}}\right)\right\}_{\rho_{\mu}<\gamma_{\mu}, \mu<\beta},
$$

where $\left\{\exists \mathfrak{r}_{\lambda} \mathfrak{A}_{\lambda}\left(\mathfrak{x}_{\lambda}\right)\right\}_{\lambda<x}$ and $\left\{\exists \mathfrak{y}_{\mu} \mathfrak{B}_{\mu}\left(\mathfrak{y}_{\mu}\right)\right\}_{\mu<\beta}$ are the sequences of all formulae in $\Delta$ and in $\Lambda$, respectively, whose outermost logical symbol is $\exists$, and $\Gamma$ and $\Theta$ are the results obtained from $\Delta$ and from $A$, respectively, by suppressing all the formulae $\exists \mathfrak{r a r}(\mathfrak{x})$ and $\exists \mathfrak{y} \mathfrak{B}(\mathfrak{y})$, and for each $\mu<\beta$, the sequence $\left\{t_{\mu, \rho}\right\}_{\rho<r_{\mu}}$ consists of all well-ordered sequences of terms whose ordinal type is identical with that of $\mathfrak{y}_{\mu}$, and each term of which is formed from the predicate symbols and the free and bound variables occurring in $\widetilde{S}$ by the help of the logical symbols. ${ }^{4)}$

5) When a sequent contains no formula having the outer-most logical symbol, the sequent is an uppermost sequent.

4) Also the $\varepsilon$-symbol is regarded as one of the logical symbols. 
A branch of $\boldsymbol{P}(\Im)$ consists of the sequents in linear sequence, passing upward within the tree structure, beginning with the lowermost sequent $\mathbb{S}$, and terminating in an uppermost sequnet or extending infinitely.

2.1. Case 1: In every branch of $\boldsymbol{P}(\Im)$, there exists at least one sequent and there exists at least one formula occurring in both the antecedent and the succedent of that sequent. By simple modifications, we obtain a formal proof of $\subseteq$ which contains no cut. Then $\subseteq$ is provable without cut.

2.2. Case 2: There exists at least one branch of $\boldsymbol{P}(\Im)$, and for every sequent occurring in that branch, there exists no formula occurring in both the antecedent and the succedent at the same time. In the rest of our proof of Theorem 4, we consider only the formulae and the sequents in that branch.

Let $D$ be the domain consisting of all of the terms, each of which is formed from those predicate symbols and free and bound variables which occur in the lowermost sequent $\mathfrak{S}$ by the help of the logical symbols. If there is not such a term at all, let $D$ be an arbitrary non-empty domain. Then, for the proof of Theorem 4, it is sufficient to show the existence of such an interpretation as makes all of our concerned sequents false with respects to $D$. This is obviously reduced to the proof of the following lemmata.

LEMMA 1. If a formula occurs in the antecedent of a sequent, then the formula does not occur in the succedent of any sequent.

LEMMA 2. If a formula $7 \mathfrak{A}$ occurs in the antecedent (or succedent) of a sequent, then the formula $\mathfrak{A}$ occurs in the succedent (or antecedent) of a sequent.

LEMMA 3. If a formula $\underset{\lambda<\alpha}{\vee} \mathfrak{A}_{\lambda}$ occurs in the antecedent (or succedent) of a sequent, then the formula $\mathfrak{A}_{\lambda}$ occurs in the antecedent (or succedent) of a sequent, for some (or every) $\lambda<\alpha$.

LEMMA 4. If a formula $\exists \mathfrak{x} \mathfrak{A}(\mathfrak{x})$ occurs in the antecedent of a sequent, then the formula $\mathfrak{A}(\varepsilon \mathfrak{E} \mathfrak{H}(\mathfrak{x}))$ occurs in the antecedent of a sequent.

LEMMA 5. If a formula $\exists \mathfrak{x} \mathfrak{A}(\mathrm{x})$ occurs in the succedent of a sequent, then the formula $\mathfrak{H}(\mathrm{t})$ occurs in the succedent of a sequent, for every sequence $t$ of elements of $D$ whose ordinal type is identical with that of $\mathfrak{x}$.

LEMMA 6. If a formula $\mathfrak{X}(\mathrm{t})$ occurs in the antecedent of a sequent, then the formula $\mathfrak{Y}(\varepsilon x \mathfrak{Y}(\mathfrak{x}))$ does not occur in the succedent of any sequent.

Lemmata 2-6 are easily proved from the structure of $\boldsymbol{P}(\widetilde{S})$. To prove Lemma 1, we shall define the concept of nesting number of formula as follows:

1) The nesting number of a prime formula is 0 .

2) The nesting number of $7 \mathfrak{A}$ is $\nu+1$, where $\nu$ is the nesting number of $\mathfrak{A}$.

3) The nesting number of $\underset{\lambda<\alpha}{\vee} \mathfrak{A}_{\lambda}$ is $\nu+1$, where $\nu$ is the supremum of the nesting numbers of $\mathfrak{Y}$ 's.

4) The nesting number of $\exists \mathfrak{x} \mathfrak{Y}(\mathfrak{x})$ is $\nu+1$, where $\nu$ is the nesting number 
of $\mathfrak{A}(\mathfrak{a})$ where $\mathfrak{a}$ is a sequence of free variables whose ordinal type is identical with that of $\mathfrak{x}$.

Proof of Lemma 1. If there were at least one formula occurring in the antecedent of a sequent and in the succedent of another sequent, there should be such a formula which has the minimum nesting number. This is contrary to the structure of $\boldsymbol{P ( \subseteq )}$, q. e.d.

The proof of the Theorem 4 and the main theorem is thus completed.

3. Finally, we shall extend the Löwenheim-Skolem thorem to the case of the predicate calculus with infinitely long expressions. For the purpose, we shall introduce the concepts 'order of a term' and 'order of a formula', defined recursively as follows:

1) The order of a free variable is 0.

2) The order of a prime formula $F \mathrm{t}$ is $\max \left\{\alpha+1, \sup _{\lambda<\alpha} \beta_{\lambda}\right\}$, where $\mathrm{t}=\left\{t_{\lambda}\right\}_{\lambda<\alpha}$ and $\beta_{\lambda}$ is the order of $t_{\lambda}$.

3) The order of $7 \mathfrak{A}$ is identical with the order of $\mathfrak{A}$.

4) The order of $\underset{\lambda<\alpha}{\vee} \mathfrak{A}_{\lambda}$ is $\max \left\{\alpha+1, \sup _{\lambda<\alpha} \beta_{\lambda}\right\}$, where $\beta_{\lambda}$ is the order of $\mathfrak{A}_{\lambda}$.

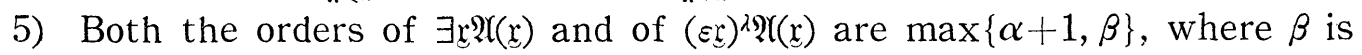
the order of $\mathfrak{R}(\mathfrak{a})$ and $\mathfrak{x}=\left\{x_{\lambda}\right\}_{k<\alpha}$.

Let $\mathfrak{F}$ be an $\varepsilon$-less formula whose order is $\alpha$ and which is satisfied in some non-empty domain, and $\subseteq$ be the sequent

$$
\mathfrak{F} \rightarrow \text {. }
$$

$\subseteq$ is, of course, unprovable in the $\varepsilon$-calculus. Let $\pi$ be the least ordinal number ${ }^{5)}$ which is strongly inaccessible with respect to $\alpha$, in the following sense:

1) If $\lambda<\pi$, then $\overline{2^{\lambda}}<\pi$, where $2^{\lambda}$ is the power set of the set consisting of all ordinal numbers $<\lambda$, and $\overline{2^{\lambda}}$ is the least ordinal number whose cardinal number is equal to that of $2^{\lambda}$.

2) If $\beta<\alpha$ and if $\alpha_{\lambda}<\pi$ for all $\lambda<\beta$, then

$$
\sup _{\lambda<\beta} \alpha_{\lambda}<\pi \text {. }
$$

Let $D$ be the domain consisting of all terms, whose order is less than or equal to $\alpha$, and each of which is formed from those predicate symbols and free or bound variables which occur in $\mathfrak{F}$. If there is not such a term at all, let $D$ be the domain consisting of an element. Then $\overline{\bar{D}} \leqq \pi$.

Now, also in the case where the treatment of Case 2 in the proof of Theorem 4 is applied to those $\subseteq$ and $D$, the proof is safely carried through. Then we have the following

EXTENDED LöWENHEIM-SKOLEM THEOREM. If an e-less formula $\mathfrak{F}$ has the order $\alpha$ and is satisfied in some non-empty domain, then there exists a non-empty

5) For example, if $\alpha \leqq \omega$, then $\pi=\omega$. 
domain $D$ such that $\mathfrak{F}$ is satisfied in $D$ and $\overline{\bar{D}} \leqq \pi$, where $\pi$ is the least ordinal number which is strongly inaccessible with respect to $\alpha$. (Clearly, $\pi$ is less than any strongly inaccenssible number which is greater than $\alpha$.)

\section{$\S 4$. Normal form theorem and interpolation theorem.}

In this section, we shall prove Craig's interpolation theorem as an application of the cut-elimination theorem. First we prove the following

NORMAL FORM THEOREM. If a sequent $\subseteq$ is provable in the $\varepsilon$-less calculus, then there exists an $\varepsilon$-less formal proof satisfying the following conditions:

1) The endsequent is 5 .

2) It contains no cut.

3) When a free variable occurs in an inference as an eigenvariable, this free variable will never occur as one of the eigenvariables in other side formula throughout the formal proof.

Proof. Let a sequent $\widetilde{S}$ be provable in the $\varepsilon$-less calculus and $\boldsymbol{P}$ be an $\varepsilon$-less formal proof which contains no cut and whose endsequent is $\widetilde{\varsigma}$. Let $\Gamma \rightarrow \Theta$ be a sequent occurring in $\boldsymbol{P}$ and $\Phi$ be a well-ordered sequence of all formulae, each of which is the side formula of a principal formula of the form $\exists \mathfrak{x} \mathfrak{A}(\mathfrak{x})$ occurring in the antecedent of a sequent that is below the sequent $\Gamma \rightarrow \Theta$. By substituting $\Gamma, \Phi \rightarrow \Theta$ for any sequent $\Gamma \rightarrow \Theta$ throughout $\boldsymbol{P}$, and by the help of simple modifications, we have a formal proof satisfying the above three conditions, q.e.d.

By using the normal form theorem, we can prove the following

INTERPOLATION THEOREM. ${ }^{6)}$ If a sequent $\mathfrak{A} \rightarrow \mathfrak{B}$ is provable in the $\varepsilon$-less calculus, then there exists a formula $\mathbb{5}$ such that both the sequents $\mathfrak{A} \rightarrow \mathfrak{S}$ and $\mathbb{\mathfrak { S }} \rightarrow \mathfrak{B}$ are provable in the e-less calculus, and those predicate symbols and free variables which occur in $\mathfrak{6}$ are all contained in both $\mathfrak{A}$ and $\mathfrak{B}$, as long as $\mathfrak{A}$ and $\mathfrak{B}$ have at least one predicate symbol in common. If the sequent $\mathfrak{A} \rightarrow \mathfrak{B}$ is provable, and if $\mathfrak{i}$ and $\mathfrak{B}$ have no predicate symbol in common, then either the sequent $\mathfrak{A} \rightarrow$ or $\rightarrow \mathfrak{B}$ is provable.

The latter half of the theorem can be proved from the first half as follows:

Let the sequent $\mathfrak{A} \rightarrow \mathfrak{B}$ be provable, and $\mathfrak{A}$ and $\mathfrak{B}$ have no predicate symbol in common. Let $P$ be a predicate symbol contained in neither $\mathfrak{A}$ nor $\mathfrak{B}$, which will be regarded as a prime formula. Then since $\mathfrak{A} \vee P \rightarrow \mathfrak{B} \vee P$ is provable, there exists a formula $\sqrt{ }$ such that both $\mathfrak{A} \vee P \rightarrow \mathfrak{}$ and $\mathfrak{} \rightarrow \mathfrak{B} \vee P$ are provable and 5 contains no predicate symbol except $P$. As 5 contains no predicate symbol except $P$, at least one of the sequents $7 P \rightarrow \mathbb{C}$ and $7 P \rightarrow 7 \Subset$ is provable. We shall consider separately the followiug two cases:

6) For the ordinary predicate calculus, this theorem had been proved in Craig [1]. 
1) Case 1: $7 P \rightarrow \mathfrak{C}$ is provable. In this case, $7 P \rightarrow \mathfrak{B} \vee P$ is provable, therefore $7 \mathfrak{B} \rightarrow \mathfrak{B} \vee \mathfrak{B}$ is provable because $\mathfrak{B}$ does not contain $P$. So the sequent $\rightarrow \mathfrak{B}$ is provable.

2) Case 2: $7 P \rightarrow 7 \mathfrak{B}$ is provable. In this case, $\mathfrak{N} \vee P \rightarrow P$ is provable, therefore $\mathfrak{A} \vee>\mathfrak{A} \rightarrow \neg \mathfrak{A}$ is provable because $\mathfrak{A}$ does not contain $P$. So the sequent $\rightarrow \mathfrak{A}$ is provable, q.e.d.

To prove the first half of the theorem, we define the degree of a normalformed proof in the sense of the normal form theorem as follows:

1) The degree of each uppermost sequent is 0 .

2) The degree of the conclusion of a logical rule of inference is $\nu+1$, provided that the degree of the conclusion of an introduction of $\exists$ in antecedent is $\nu+2$, where $\nu$ is the degree of the premise or the supremum of all degrees of the premises.

3) The degree of the conclusion of a structural rule of inference is identical with the degree of the premise.

4) The degree of a normal-formed proof is the degree of the endsequent.

Our proof is now reduced to the proof of the following

TheOREM 5. If a sequent $\Gamma_{1}, \Gamma_{2} \rightarrow \Theta_{1}, \Theta_{2}$ is provable in the e-less calculus, and if the sequents $\Gamma_{1} \rightarrow \Theta_{1}$ and $\Gamma_{2} \rightarrow \Theta_{2}$ have at least one predicate symbol in common, then there exists a formula (5 such that both the sequents

$$
\Gamma_{1} \rightarrow \text { (5, } \Theta_{1} \quad \text { and } \quad \Gamma_{2},(5) \Theta_{2}
$$

are provable and those predicate symbols and free variables which occur in 5 are all contained in both $\Gamma_{1} \rightarrow \Theta_{1}$ and $\Gamma_{2} \rightarrow \Theta_{2}$.

This theorem is proved by transfinite induction on the degree $\delta$ of the normal-formed proof whose endsequent is $\Gamma_{1}, \Gamma_{2} \rightarrow \Theta_{1}, \Theta_{2}$. We divide several cases.

Case 1: $\delta=0$. The theorem is obvious.

Case 2: $\delta>0$ and the lowermost logical rule of inference of the formal proof containing no cut whose endsequent is $\Gamma_{1}, \Gamma_{2} \rightarrow \Theta_{1}, \Theta_{2}$ is

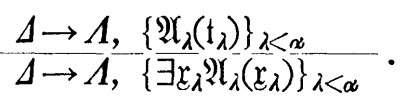

There exists a normal-formed proof whose degree is less than $\delta$ and whose endsequent is $\Gamma_{1}^{\#}, \Gamma_{1}, \Gamma_{2}^{*}, \Gamma_{2} \rightarrow \Theta_{1}^{*}, \Theta_{2}^{*}$, where

1) $\Theta^{*}$ is the result obtained from $\Theta$ by substituting $\mathfrak{A}(\mathrm{t})$ for every $\exists \mathfrak{x} \mathfrak{A}(\mathfrak{x})$ throughout $\Theta$, and

2) $\Gamma^{*}$ is the sequence of all formulae

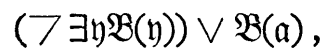

where each $\exists \mathfrak{y} \mathfrak{B}(\mathfrak{y})$ is a principal formula of an introduction of $\exists$ in antecedent and has a descendant in $\Gamma$ of the endsequent $\Gamma_{1}, \Gamma_{2} \rightarrow \Theta_{1}, \Theta_{2}$, and $\mathfrak{B}(\mathfrak{a})$ is the 
side formula of that principal formula $\exists \mathfrak{y} \mathfrak{B}(\mathfrak{y})$, and at least one eigenvariable in $\mathfrak{a}$ is contained in $\left\{\mathrm{t}_{\lambda}\right\}_{\lambda<a}$.

By the hypothesis of the transfinite induction on the degree, there exists a formula $\mathfrak{S}^{\prime}\left(\mathfrak{b}, \mathfrak{c}_{1}, \mathfrak{c}_{2}\right)$, such that both $\Gamma_{1}^{\#}, \Gamma_{1} \rightarrow \mathfrak{c}^{\prime}\left(\mathfrak{b}, \mathfrak{c}_{1}, \mathfrak{c}_{2}\right), \Theta_{1}^{*}$ and $\Gamma_{2}^{\#}, \Gamma_{2}, \mathfrak{C}^{\prime}\left(\mathfrak{b}, \mathfrak{c}_{1}, \mathfrak{c}_{2}\right)$ $\rightarrow \Theta_{2}{ }^{*}$ are provable and those predicate symbols and free variables which occur in $\mathfrak{S}^{\prime}\left(\mathfrak{b}, \mathfrak{c}_{1}, \mathfrak{c}_{2}\right)$ are all contained in both $\Gamma_{1}{ }^{\sharp}, \Gamma_{1} \rightarrow \Theta_{1}^{*}$ and $\Gamma_{2}^{\sharp}, \Gamma_{2} \rightarrow \Theta_{2}^{*}$, where $\mathfrak{b}$ is the sequence of those free variables which are contained in neither a's nor $\Gamma_{1}, \Gamma_{2} \rightarrow \Theta_{1}, \Theta_{2}$, and $\mathfrak{c}_{1}$ (or $\mathfrak{c}_{2}$ ) is the sequence of those free variables which occur in one of the a's and which are contained in both $\Gamma_{1}^{\#}$ and $\Theta_{2}^{*}$ (or in both $\Gamma_{2}^{*}$ and $\left.\Theta_{1}^{*}\right)$. Then both the sequent

and

$$
\begin{aligned}
& \Gamma_{1}^{\sharp}, \Gamma_{1} \rightarrow \exists \mathfrak{q}_{1}>\exists \mathfrak{q}_{2}>\left(\mathfrak{S}^{\prime}\left(\mathfrak{b}, \mathfrak{q}_{1}, \mathfrak{q}_{2}\right), \Theta_{1}\right. \\
& \Gamma_{2}^{\sharp}, \Gamma_{2}, \exists \mathfrak{q}_{1}>\exists \mathfrak{q}_{2}>\mathfrak{S}^{\prime}\left(\mathfrak{h}, \mathfrak{q}_{1}, \mathfrak{q}_{2}\right) \rightarrow \Theta_{2}
\end{aligned}
$$

are provable, and then, since every sequent

$$
\rightarrow \exists \mathfrak{z}[(7 \exists \mathfrak{y} \mathfrak{B}(\mathfrak{y})) \vee \mathfrak{B}(\mathfrak{z})]
$$

is provable, both the sequents

and

$$
\begin{aligned}
& \Gamma_{1} \rightarrow \exists \mathfrak{q}_{1}>\exists \mathfrak{q}_{2}>\mathfrak{S}^{\prime}\left(\mathfrak{b}, \mathfrak{q}_{1}, \mathfrak{q}_{2}\right), \Theta_{1} \\
& \Gamma_{2}, \exists \mathfrak{q}_{1}>\exists \mathfrak{q}_{2}>\mathfrak{S}^{\prime}\left(\mathfrak{b}, \mathfrak{q}_{1}, \mathfrak{q}_{2}\right) \rightarrow \Theta_{2}
\end{aligned}
$$

are provable. Hence, required formula ${ }^{5}$ is, for example,

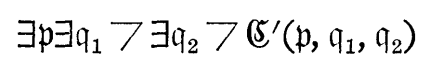

Case 3: $\delta>0$ and the lowermost logical rule of inference of the formal proof is

$$
\frac{\left\{\mathfrak{P}_{\lambda}\left(\mathfrak{a}_{\lambda}\right)\right\}_{\lambda<\alpha}, \Delta \rightarrow \Lambda}{\left\{\exists \mathfrak{x}_{\lambda} \mathfrak{A}_{\lambda}\left(\mathfrak{x}_{\lambda}\right)\right\}_{\lambda<\alpha}, \Delta \rightarrow \Lambda} .
$$

There exists a formal proof containing no cut whose degree is less than $\delta$ and whose endsequent is $\Gamma_{1}^{*}, \Gamma_{2}{ }^{*} \rightarrow \Theta_{1}, \Theta_{2}$ where each $\Gamma^{*}$ is the result obtained from $\Gamma$ by substituting $\mathfrak{A}(\mathfrak{a})$ for every principal formula $\exists \mathfrak{x} \mathfrak{A}(\mathfrak{x})$ throughout $\Gamma$. By the hypothesis of the transfinite induction of the degree, there exists a formula $\sigma^{\prime}$ such that both the sequents

$$
\Gamma_{1}^{*} \rightarrow \mathbb{(}^{\prime}, \Theta_{1} \quad \text { and } \Gamma_{2}^{*}, \boldsymbol{c}^{\prime} \rightarrow \Theta_{2}
$$

are provable and those predicate symbols and free variables which occur in (5' are all contained in both $\Gamma_{1}{ }^{*} \rightarrow \Theta_{1}$ and $\Gamma_{2}{ }^{*} \rightarrow \Theta_{2}$. Here we can assume, without loss of generality, that $\Gamma_{1}$ and $\Gamma_{2}$ have no formula in common. Then required (5) is (5) itself.

Other cases. The treatment is similar to the above, q.e.d. 


\section{$\S 5$. Karp's completeness theorem.}

In [5] Karp announced a completeness theorem for the first-order predicate calculus with infinitely long expressions; she proposed thereby a system with the usual form of restriction on eigenvariables. In this section, we shall give a formal system which is essentially identical with Karp's calculus, and shall prove that the new system is equivalent to the calculus formulated in $\S 2$. This equivalence proof will be carried through by the same idea as in Maehara [7].

In what follows, we shall use notations of the form $\forall \mathfrak{x} \mathfrak{Y}(\mathfrak{x})$ as an abbreviation of $7 \exists \mathfrak{x}>\mathfrak{A}(\mathfrak{x})$.

In the new calculus, we use the postulates for the original calculus explained in $\S 2$, as they are, except the introduction of $\exists$ in antecedent, and

$$
\forall \exists \text {-rule : } \frac{\left\{\mathfrak{A}_{\lambda}\left(\mathfrak{t}_{\lambda}, \mathfrak{a}_{\lambda}\right)\right\}_{\lambda<\alpha}, \Gamma \rightarrow \Theta}{\left.\left\{\forall \mathfrak{x}_{\lambda} \exists \mathfrak{y}\right)_{\lambda} \mathfrak{A}_{\lambda}\left(\mathfrak{x}_{\lambda}, \mathfrak{y}_{\lambda}\right)\right\}_{\lambda<\alpha}, \Gamma \rightarrow \Theta},
$$

where, all the free variables contained in a's shall not occur in the conclusion. For each variable contained in a's or t's, an ordinal number named the height can be uniquely defined as follows:

The height of a free variable in $a_{\lambda}$ is $\nu+1$, where $\nu$ is the supremum of the heights of all free variables in $t_{\lambda}$. If a free variable is not contained in any $\mathfrak{a}$, the height of the free variable is 0 .

As the introduction of $\exists$ in antecedent we use the $\forall \exists$-rule with empty sequences of universal quantifiers standing at the front.

The above-mentioned new calculus will be called ' $\forall \exists$-calculus', and the original calculus explained in $\S 2$ ' $\varepsilon$-less calculus' as before.

THEOREM 1. If a sequent is provable in the e-less calculus, then it is provable in the $\forall \exists$-calculus.

COROLLARY 1. Every $\varepsilon$-less sequent which is valid in any non-empty domain is provable in the $\forall \exists$-calculus (Completeness theorem for the $\forall \exists$-calculus).

COROLlARY 2. Every $\varepsilon$-less sequent which is provable in the $\varepsilon$-lcalculus is provable in the $\forall \exists$-calculus (The second $\varepsilon$-theorem for the $\forall \exists$-calculus).

Proof of Theorem 1. Let a sequent $\Gamma \rightarrow \Theta$ be provable in the $\varepsilon$-less calculus, and $\boldsymbol{P}$ be a formal proof in the sense of the $\varepsilon$-less calculus, whose endsequent is $\Gamma \rightarrow \Theta$. Let a sequence

$$
\left\{\exists \mathfrak{x}_{\lambda} \mathfrak{R}_{\lambda}\left(\mathrm{t}_{\lambda}, \mathfrak{x}_{\lambda}\right)\right\}_{\lambda<\infty}
$$

consist of all principal formulae of the inferences 'the introduction of $\exists$ in antecedent' which are contained in $\boldsymbol{P}$, and $t_{\lambda}$ be the sequence of all terms occurring in $\exists \mathfrak{x}_{,}, \mathfrak{A}_{\lambda}\left(\mathrm{t}_{\lambda}, \mathfrak{x}_{\lambda}\right)(\lambda<\alpha)$. Then the sequent 


$$
\left\{\left(7 \exists \mathfrak{x}_{\lambda} \mathfrak{U}_{\lambda}\left(\mathrm{t}_{\lambda}, \mathfrak{x}_{\lambda}\right)\right) \vee \mathfrak{A}_{\lambda}\left(\mathrm{t}_{\lambda}, \mathfrak{a}_{\lambda}\right)\right\}_{\lambda<\alpha}, \Gamma \rightarrow \Theta
$$

is provable without $\forall \exists$-rule in the $\forall \exists$-calculus, where $a$ 's are the sequences of the eigenvariables corresponding to those principal formula.

Accordingly, the sequent

$$
\left\{\forall \mathfrak{y}_{\lambda} \exists \exists_{\lambda}\left[\left(7 \mathfrak{x}_{\lambda} \mathfrak{A}_{\lambda}\left(\mathfrak{y}_{\lambda}, \mathfrak{x}_{\lambda}\right)\right) \vee \mathfrak{A}_{\lambda}\left(\mathfrak{y}_{\lambda}, \mathfrak{z}_{\lambda}\right)\right]\right\}_{\lambda<a}, \Gamma \rightarrow \Theta
$$

is provable in the $\forall \exists$-calculus, by the help of the $\forall \exists$-rule. On the other hand, the sequents

$$
\rightarrow \forall \mathfrak{y}_{\lambda} \exists \gamma_{\lambda}\left[\left(7 \exists \mathfrak{x}_{\lambda} \mathfrak{A}_{\lambda}\left(\mathfrak{y}_{\lambda}, \mathfrak{x}_{\lambda}\right)\right) \vee \mathfrak{A}_{\lambda}\left(\mathfrak{y}_{\lambda}, \mathfrak{\gamma}_{\lambda}\right)\right] \quad(\lambda<\alpha)
$$

are all provable in the $\forall \exists$-calculus. Hence the sequent $\Gamma \rightarrow \Theta$ is provable in the $\forall \exists$-calculus, q.e.d.

THEOREM 2. If a sequent is provable in the $\forall \exists$-calculus, then it is provable in the $\varepsilon$-less calculus.

PROOF. Let a formal proof in the sense of the $\forall \exists$-calculus be given. Replace each inference of the form

$$
\frac{\left\{\mathfrak{A}_{\lambda}\left(\mathfrak{t}_{\lambda}, \mathfrak{a}_{\lambda}\right)\right\}_{\gamma_{<\nu}, \Gamma \rightarrow \Theta}}{\left\{\forall \mathfrak{c}_{\lambda} \exists \mathfrak{y}_{\lambda} \mathfrak{A} \mathfrak{H}_{\lambda}\left(\mathfrak{x}_{\lambda}, \mathfrak{y}_{\lambda}\right)\right\}_{\lambda<\boldsymbol{x}}, \Gamma \rightarrow \Theta}
$$

by the following figure:

$$
\begin{gathered}
\left\{\mathfrak{H}_{\lambda}\left(\mathfrak{t}_{\lambda}, \mathfrak{a}_{\lambda}\right)\right\}_{\lambda<\alpha}, \Gamma \rightarrow \Theta \\
\hline\left\{\exists \mathfrak{y} \mathfrak{y}_{\lambda}\left(\mathfrak{t}_{\lambda}, \mathfrak{y}_{\lambda}\right)\right\}_{\lambda<\alpha}, \Gamma \rightarrow \Theta \\
\hline \Gamma \rightarrow \Theta,\left\{7 \exists \mathfrak{y} \mathfrak{y}_{\lambda}\left(\mathfrak{t}_{\lambda}, \mathfrak{y}_{\lambda}\right)\right\}_{\lambda<\alpha} \\
\hline \Gamma \rightarrow \Theta,\left\{\exists \mathfrak{x}_{\lambda}>\exists \exists \mathfrak{y}_{\lambda} \mathfrak{I}_{\lambda}\left(\mathfrak{x}_{\lambda}, \mathfrak{y}_{\lambda}\right)\right\}_{\lambda<\alpha} \\
\hline\left\{\forall \mathfrak{x}_{\lambda} \exists \mathfrak{y}_{\lambda} \mathfrak{\mathfrak { P } _ { \lambda }}\left(\mathfrak{x}_{\lambda}, \mathfrak{y}_{\lambda}\right)\right\}_{\lambda<\alpha}, \Gamma \rightarrow \Theta
\end{gathered}
$$

Then we have a formal proof in the sense of the $\varepsilon$-less calculus, which has the same endsequent, q. e.d.

$$
\begin{gathered}
\text { School of Science and Engineering, } \\
\text { Waseda University, Tokyo and } \\
\text { Tokyo University of Education, Tokyo }
\end{gathered}
$$

\section{References}

[1] W. Craig, Linear reasoning. A new form of the Herbrand-Gentzen theorem, J. Symbolic Logic, 22 (1957), 250-268.

[2] G. Gentzen, Untersuchungen über das logische Schließen, Math. Z., 39 (1935), 176-210, 405-431.

[3] K. Gödel, Die Vollständigkeit der Axiome des logischen Funktionenkalküls, Monatsh. f. Math. Phys., 37 (1930), 349-360.

[4] D. Hilbert and P. Bernays, Gundlangen der Mathematik, Bd. 2, Berlin, 1939.

[5] C. Karp, Independence proofs in predicate logic with infinitely long expressions. Lecture in International Congress for Logic, Methodology and Philosophy of Science 1960, Stanford. 
[6] S. C. Kleene, Introduction to metamathematics, Amsterdam-Groningen, 1952.

[7] S. Maehara, The predicate calculus with $\varepsilon$-symbol, J. Math. Soc. Japan, 7 (1955), 323-344.

[8] K. Schütte, Ein System des verknüpfen den Schließens, Archiv f. math. Logik u. Grundlagenforschung 2 (1956), 55-67.

[9] D. Scott and A. Tarski, The sentential calculus with infinitely long expressions, Colloq. Math., 6 (1958), 165-170. 